\title{
Dexmedetomidine suppresses sevoflurane anesthesia-induced neuroinflammation through activation of the PI3K/Akt/mTOR pathway
}

Nan Wang and Mingyu Wang ${ }^{*}$ (D)

\begin{abstract}
Background: Sevoflurane, an inhalational general anesthetic, has become one of the most widely used inhalational anesthetics in surgery. However, previous studies have found that sevoflurane anesthesia can trigger an inflammatory response, resulting in secondary damage. Dexmedetomidine (DEX), a highly-selective a adrenergic receptor agonist, is widely used as an anesthetic adjuvant in the clinic. In this study we investigated whether DEX was able to suppress sevoflurane-induced neuroinflammation.

Methods: The aim was to determine the mechanism of action of the suppressive effect of DEX using a rat model. Rats were randomly divided into a control group $(n=10)$, low-dose sevoflurane group (L-Sev; $n=10)$, high-dose sevoflurane group (H-Sev; $n=10)$, vehicle group $(n=10)$, DEX group $(n=10)$ and DEX + LY294002 (a specific inhibitor of PI3K) group $(n=10)$. The rats in vehicle, DEX and DEX + LY294002 groups were in the presence of highdose sevoflurane exposure. Western blotting was used to measure the expression of proinflammatory cytokines (IL6, IL-8, TNF-a) and the activity level of the phosphatidylinositol 3-hydroxy kinase/protein kinase B/mammalian target of rapamycin (PI3K/Akt/mTOR) pathway.

Results: We found that sevoflurane anesthesia induced an increase in the levels of pro-inflammatory cytokines, while decreasing activation of the PI3K/Akt/mTOR pathway in both the cortex and hippocampus of rats. Treatment with DEX reduced pro-inflammatory cytokine levels and prevented inactivation of the PI3K/Akt/mTOR pathway. Moreover, LY294002, an inhibitor of the PI3K/Akt/mTOR pathway, reduced the anti-inflammatory activity of DEX.

Conclusions: These data suggest that the PI3K/Akt/mTOR pathway contributes to sevoflurane-induced neuroinflammation and that activation of PI3K/Akt/mTOR signaling by DEX could help reduce the neuroinflammatory effects of sevoflurane.
\end{abstract}

Keywords: Dexmedetomidine, Sevoflurane, Neuroinflammation, PI3K/Akt/mTOR pathway

\section{Background}

In recent years the inhalational anesthetic sevoflurane has replaced other volatile anesthetics due to its quick induction, stable maintenance, rapid revival and because the irritation it causes is mild [1]. However, recent evidence has shown that sevoflurane can lead to pathophysiological alterations of the brain during the recovery period, such as

\footnotetext{
*Correspondence: mingyuwang1211@163.com

Department of Anesthesiology, Cancer Hospital of China Medical University,

Liaoning Cancer Hospital \& Institute, Dalian Medical University Clinical

Oncology College, Shenyang 110042, Liaoning, China
}

neuronal apoptosis, tau aggresomes, abnormal discharge of neurons, and neuroinflammation, which cause neurodegenerative changes in the development of the mammalian brain [2-6]. Exposure to anesthesia in childhood may lead to adverse neurodevelopmental outcomes in children [7]. Despite evidence linking inhalational anesthesia to neurodegenerative effects, it is still considered safer than other methods of anesthesia, and more than 3 million children undergo inhaled anesthesia every year $[8,9]$. Therefore, there is an urgent

(c) The Author(s). 2019 Open Access This article is distributed under the terms of the Creative Commons Attribution 4.0 International License (http://creativecommons.org/licenses/by/4.0/), which permits unrestricted use, distribution, and 
need to find anesthetic adjuvants that reduce the neurotoxicity of inhaled anesthetics.

Dexmedetomidine (DEX), a selective $\alpha 2$-adrenoceptor agonist, is used intravenously as a sedative or adjuvant of local anesthetics used in peripheral nerve blocks, where it prolongs the duration of sensory and motor blockades without causing toxicity [10-12].In addition, DEX is able to cross the blood brain barrier and stimulate $\alpha 2$-adrenoceptors centrally and has been shown to exert a neuroprotective effect [13]. Wang et al. found that treatment with DEX could inhibit the expression of inflammatory cytokines and their mediators, resulting in reduced focal cerebral ischemia-reperfusion injury in rats. These data suggest that inhibition of the nuclear factor- $\kappa B$ pathway (NF- $\kappa B$ ) may be a mechanism underlying the neuroprotective action of DEX [14]. Treatment with DEX after subarachnoid haemorrhage (SAH) attenuated SAH-induced early brain injury, partially through suppression of the toll like receptor 4 (TLR4)/NF-kB pathway and the NLRP3 inflammasome [15]. Additionally, DEX has been shown to improve post-operative cognitive dysfunction in aging mice by inhibition of the hippocampal inflammatory response and reduction of neuronal apoptosis [16]. Taken together, these data suggest that DEX has anti-inflammatory properties in the central nervous system (CNS).

Given the inflammation induced by sevoflurane and the anti-inflammatory properties of DEX, we aimed to ascertain whether DEX could provide protection from sevoflurane anesthesia-induced inflammation in the CNS and ascertain whether these protective effects were closely associated with the phosphatidylinositol 3-hydroxy kinase/protein kinase $\mathrm{B} /$ mammalian target of rapamycin (PI3K/Akt/mTOR) pathway.

\section{Methods}

\section{Animals}

Fifty healthy Sprague-Dawley (SD) rats, weighing 150$200 \mathrm{~g}$ were purchased from the Animal Experiment Center of the Institute of Radiation Medicine of the Chinese Academy of Medical Sciences, China. The rats were fed food and water under specific pathogen-free conditions and housed in a $12 \mathrm{~h} \mathrm{light/dark}$ cycle at $22-24{ }^{\circ} \mathrm{C}$. All animal procedures were approved by the Institute of Radiation Medicine of the Chinese Academy of Medical Sciences and conducted in accordance with the ethical principles for Experiments on Animals, in addition to international standards.

\section{Groups and treatments}

Rats were randomly divided into six groups: control group $(n=10)$, low-dose sevoflurane group (L-Sev; $\mathrm{n}=$ $10)$, high-dose sevoflurane group $(\mathrm{H}-\mathrm{Sev} ; \mathrm{n}=10)$, vehicle group $(\mathrm{n}=10)$, DEX group $(\mathrm{n}=10)$ and DEX +
LY294002 (a specific inhibitor of PI3K) group $(n=10)$. All rats were placed in an anesthesia induction chamber and received anesthesia using an inhalation machine. Oxygen concentration and anesthesia doses were continuously monitored. The control group was treated with $60 \% \mathrm{O}_{2}$ for $2 \mathrm{~h}$, the L-Sev group with $1.5 \%$ sevoflurane inhalation for $2 \mathrm{~h}$ and the remaining groups $(\mathrm{H}-\mathrm{Sev}$ group, vehicle group, DEX group and DEX + LY294002 group) were treated with $3 \%$ sevoflurane inhalation for $2 \mathrm{~h}$ [17]. One hour prior to sevoflurane treatment, the vehicle group received an intraperitoneal injection of saline, the DEX group received an intraperitoneal injection of $4 \mu \mathrm{g} / \mathrm{kg}$ DEX [18] and the DEX + LY294002 group received an intraperitoneal injection of $4 \mu \mathrm{g} / \mathrm{kg}$ DEX and intracerebroventricular injection of $25 \mu \mathrm{g} / 5 \mu \mathrm{l}$ LY294002 (Sigma-Aldrich Chemical Company, USA) [19]. The time at which anesthesia commenced was when sevoflurane concentration had reached a maximum for each group. Gas flow in the anesthesia chamber was maintained at a rate of $4 \mathrm{~L} / \mathrm{min}$.

\section{Tissue preparation}

At the end of the experiment, rats were sacrificed under anesthesia using $50 \mathrm{mg} / \mathrm{kg}$ sodium pentobarbital by intraperitoneal injection. The brain tissue was removed and a subset of brain samples used to prepare $10 \mu \mathrm{m}$ sections for use in immunofluorescence staining. The remaining brains were placed on Aluminum foil and the cortical and the hippocampal tissues separated then stored at $-80^{\circ} \mathrm{C}$ for Western blot analysis.

\section{Western blot analysis}

Cortical and hippocampal tissue samples were lysed and total protein extracted using whole-cell protein extraction kits (Beyotime, China) in accordance with the manufacturer's protocols. A bicinchoninic acid (BCA) protein quantification kit (Beyotime, China) was used to measure protein concentration in the samples. Proteins were separated using 10\% SDSPAGE then transferred to polyvinylidene difluoride (PVDF) membranes. The membranes were blocked using 2\% BSA in TBST at room temperature for $2 \mathrm{~h}$. Membranes were then incubated with primary antibodies overnight at $4{ }^{\circ} \mathrm{C}$. The primary antibodies used in our study included rabbit anti-IL-6 (1:1000, Abcam, USA), IL-8 (1:1000, Abcam, USA), TNFa (1: 1000, Abcam, USA), PI3K (1:1000, CST, USA), pPI3K (1:1000, CST, USA), Akt (1:1000, CST, USA), p-Akt (1:1000, CST, USA), mTOR (1:1000, CST, USA), p-mTOR (1:1000, CST, USA) and GAPDH (1: 4000, Proteintech, USA). Following staining with a primary antibody, three TBST washes were preformed and the membranes were then incubated with a horseradish peroxidase-conjugated goat anti- 
rabbit IgG secondary antibody (1:4000, Proteintech, USA) for $2 \mathrm{~h}$ at room temperature. PVDF membranes were then washed three times with TBST and developed using an ECL Plus reagent. The optical density of each protein was analyzed using Image J software (National Institutes of Health, USA) and was standardized to GAPDH or corresponding total protein.

\section{Statistical analysis}

All values are presented as means \pm SD. Comparisons between groups were conducted using $\mathrm{t}$-tests and oneway ANOVA followed by a post hoc Tukey test. Differences were considered statistically significant at a level of $P<0.05$.

\section{Results}

Sevoflurane induced neuroinflammation in rats

To determine the effect of sevoflurane on neuroinflammation, expression levels of the inflammatory markers IL-6, IL-8 and TNF- $\alpha$ were measured in the cortex and hippocampus of rats in the control, L-Sev and $\mathrm{H}-\mathrm{Sev}$ groups by Western blotting. IL-6, IL- 8 and TNF- $\alpha$ expression increased significantly in the cortex and hippocampus after low or high-dose sevoflurane anesthesia compared with that of the control group (Fig. 1, $p<0.05$ ). Moreover, the high-dose sevoflurane group exhibited significantly higher levels of IL-6, IL8 and TNF- $\alpha$ than the low-dose group, indicating that the induction of inflammation was dose-dependent (Fig. 1, $p<0.05$ ).

Sevoflurane inhibited the PI3K/Akt/mTOR pathway in rats The levels of PI3K, Akt and mTOR expression were assayed, in addition to their phosphorylation status, in the cortex and hippocampus of rats from the control, L-Sev, and $\mathrm{H}-\mathrm{Sev}$ groups in order to assess the effect of sevoflurane anesthesia on PI3K/Akt/mTOR signaling. As shown in Fig. 2, both low and high-dose sevoflurane treatment groups exhibited significantly decreased levels of PI3K/Akt/

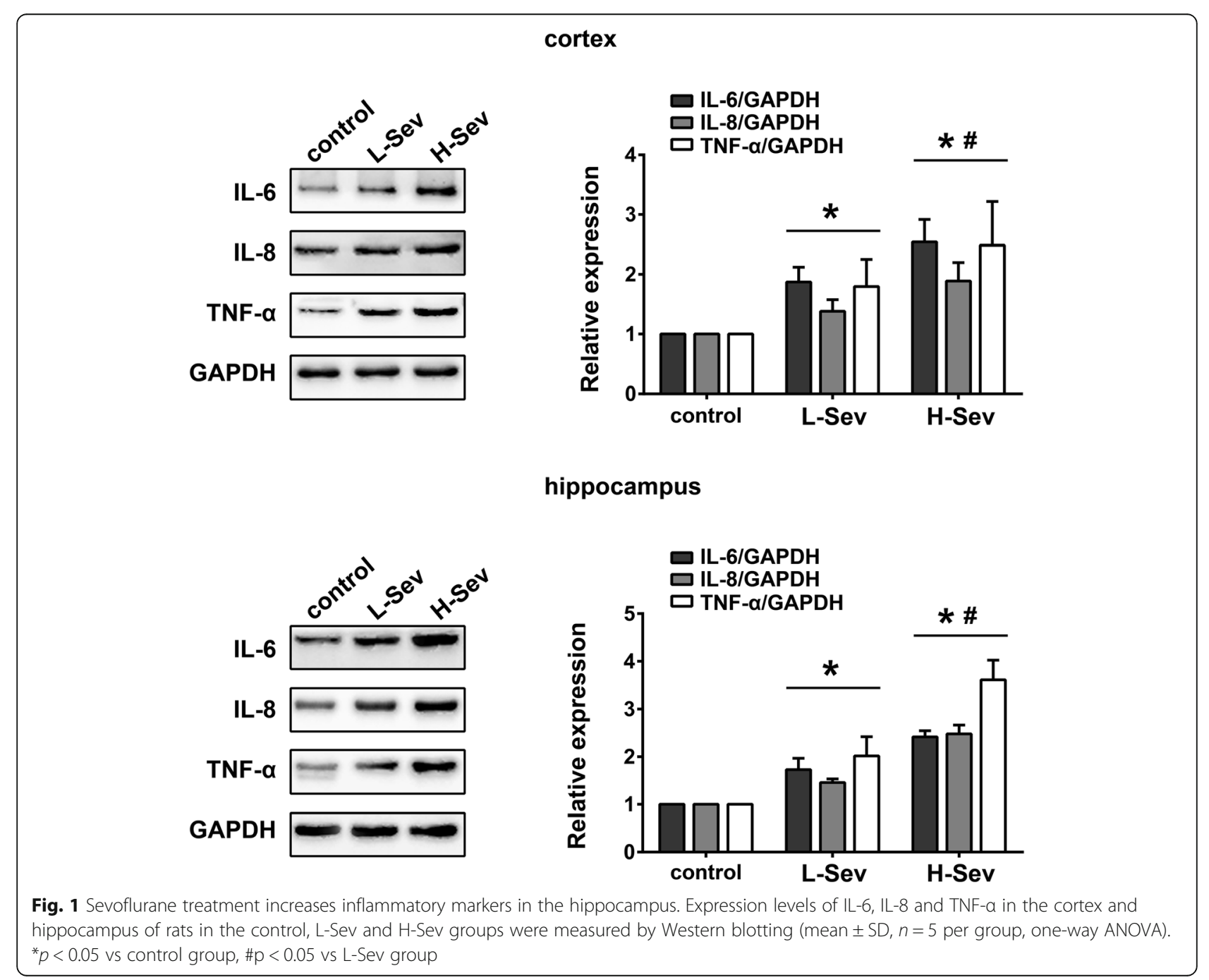


cortex

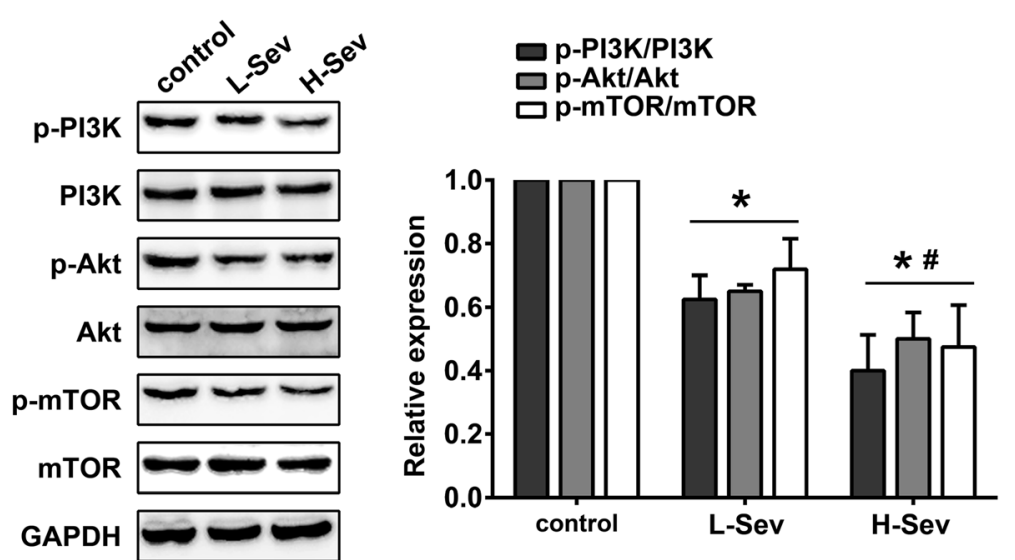

hippocampus
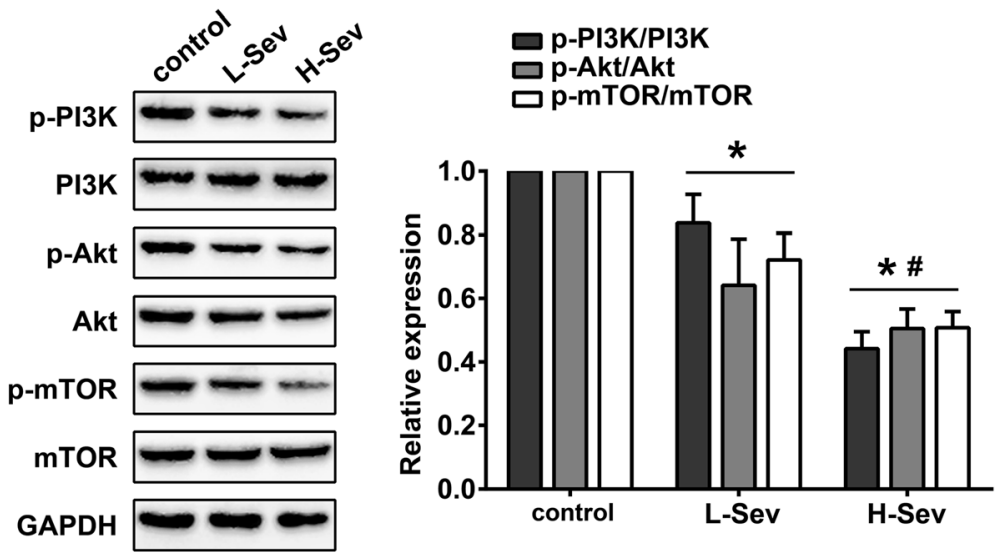

Fig. 2 Sevoflurane inhibited the PI3K/Akt/mTOR pathway in rats. Cortical and hippocampal levels of PI3K, p-PI3K, Akt, p-Akt, mTOR and p-mTOR of rats in the control, L-Sev and H-Sev groups were measured by Western blotting (mean \pm SD, $n=5$ per group, one-way ANOVA). ${ }^{*} p<0.05$ vs control group, \#p<0.05 vs L-Sev group

mTOR pathway phosphorylation $(p<0.05)$. Moreover, the level of PI3K/AKT/mTOR pathway activity was significantly lower in the cortex and hippocampus after high-dose sevoflurane anesthesia compared with that in the L-Sev group (Fig. 2, $p<0.05$ ).

\section{Dexmedetomidine suppressed sevoflurane-induced neuroinflammation in rats}

To determine if dexmedetomidine could reduce neuroinflammation induced by sevoflurane anesthesia, the levels of IL- 6 , IL- 8 and TNF- $\alpha$ were measured in the cortex and hippocampus of rats in the vehicle and DEX groups. Rats treated with DEX exhibited decreased levels of IL- 6 , IL- 8 and TNF- $\alpha$ compared with the vehicle control group, suggesting that DEX can inhibit sevoflurane anesthesia-induced neuroinflammation (Fig. 3, $p<0.05$ ).
Dexmedetomidine activated the PI3K/Akt/mTOR pathway in sevoflurane-treated rats

Using Western blotting, we assayed the effects of DEX on PI3K/Akt/mTOR pathway activation. As shown in Fig. 4, the levels of PI3K, Akt and mTOR phosphorylation increased significantly in the cortex and hippocampus in the DEX group compared with the vehicle group, indicating that DEX could activate the PI3K/Akt/mTOR pathway under sevoflurane anesthesia $(\mathrm{p}<0.05)$.

Blockade of the PI3K/Akt/mTOR pathway reduced the anti-inflammatory activity of dexmedetomidine

Finally, to determine whether the PI3K/Akt/mTOR pathway is involved in the anti-inflammatory effects of DEX, rats were treated with DEX in the presence or absence of LY294002 and the expression of IL-6, IL-8 and TNF- $\alpha$ in the cortex and hippocampus assessed by Western blot analysis. As shown in Fig. 5, the levels of 


\section{cortex}
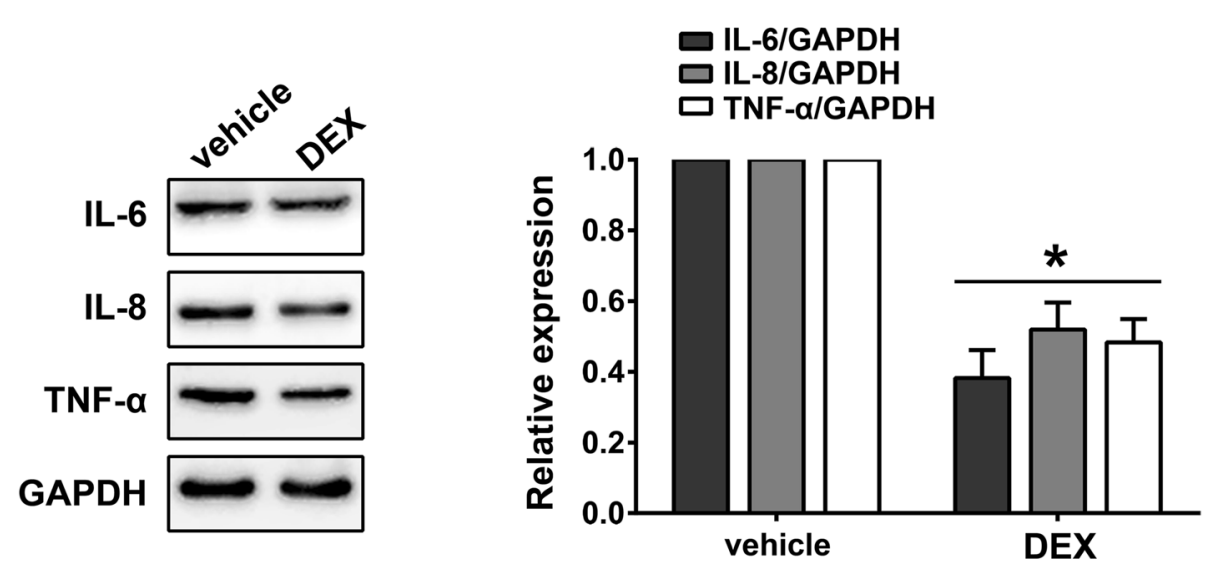

hippocampus
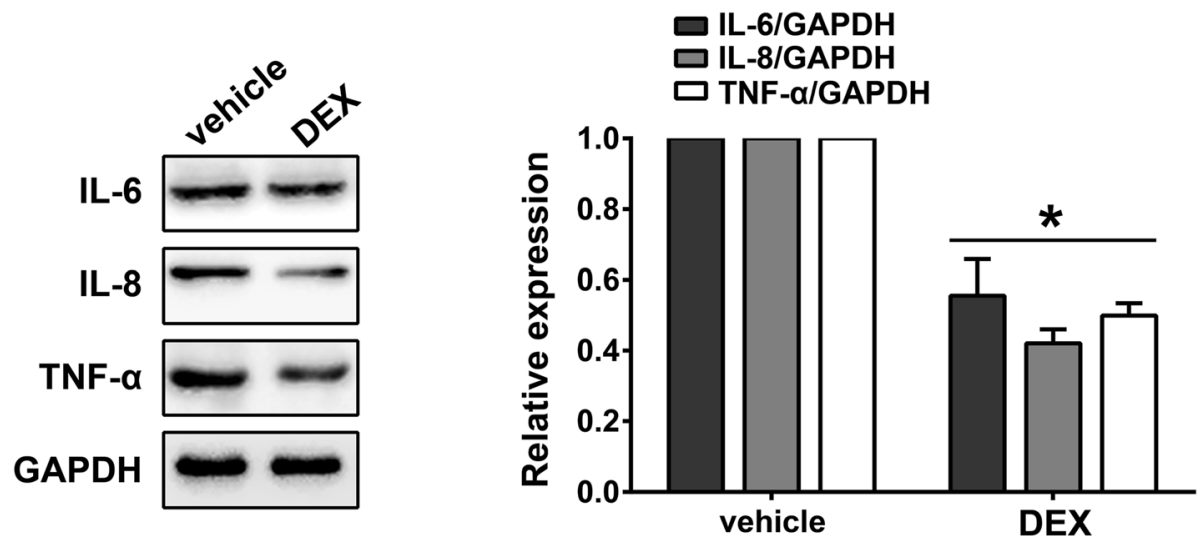

Fig. 3 Dexmedetomidine suppressed sevoflurane-induced neuroinflammation in rats Western blot measurements of IL-6, IL-8 and TNF- a from cortical and hippocampal samples of DEX and vehicle-treated rats (mean $\pm \mathrm{SD}, n=5$ per group, t test). ${ }^{*} p<0.05$ vs vehicle group

IL-6, IL-8 and TNF- $\alpha$ increased significantly in the cortex and hippocampus in the DEX+LY294002 group compared with the DEX group, indicating that LY294002 reduced the anti-inflammatory activity of DEX under sevoflurane anesthesia $(p<0.05)$.

\section{Discussion}

In the present study, we observed that sevoflurane anesthesia induced an increase in the expression of proinflammatory factors and a decrease in PI3K/Akt/mTOR pathway activity in both the cortex and hippocampus of rats. Additionally, we found that DEX treatment could restore PI3K/Akt/mTOR activity in rats treated with sevoflurane anesthesia and blockade of the PI3K/Akt/ mTOR pathway reduced the anti-inflammatory activity of DEX. Thus, we propose that DEX can suppress sevoflurane anesthesia-induced neuroinflammation by modulating PI3K/Akt/mTOR pathway activity.

Postoperative neuroinflammation is a common pathological phenomenon in the CNS, which can lead to secondary damage, such as delirium, cognitive dysfunction, Alzheimer's disease and additional detrimental effects $[20,21]$. A healthy immune response is crucial to ensure proper wound healing and repair of tissue damage, in addition to combating infection without harming the host's own cells or tissues. In contrast, an excessive inflammatory response is harmful and can result in severe tissue damage and even death [20, 22]. Inhalational anesthetics, such as isoflurane and sevoflurane, can trigger pathological inflammatory responses during surgery. Isoflurane, which has been used since the $1980 \mathrm{~s}$, is metabolized slowly leading to reduced induction of anesthesia during surgery and shorter recovery times [23]. The use of sevoflurane began a decade later. It has a lower blood-gas partition coefficient than other anesthetics, leading to rapid induction of anesthesia and faster recovery times after anesthesia $[24,25]$. Several decades of research have been conducted on the toxicity and side effects of these volatile anesthetics. Here, we investigated the relationship 
cortex
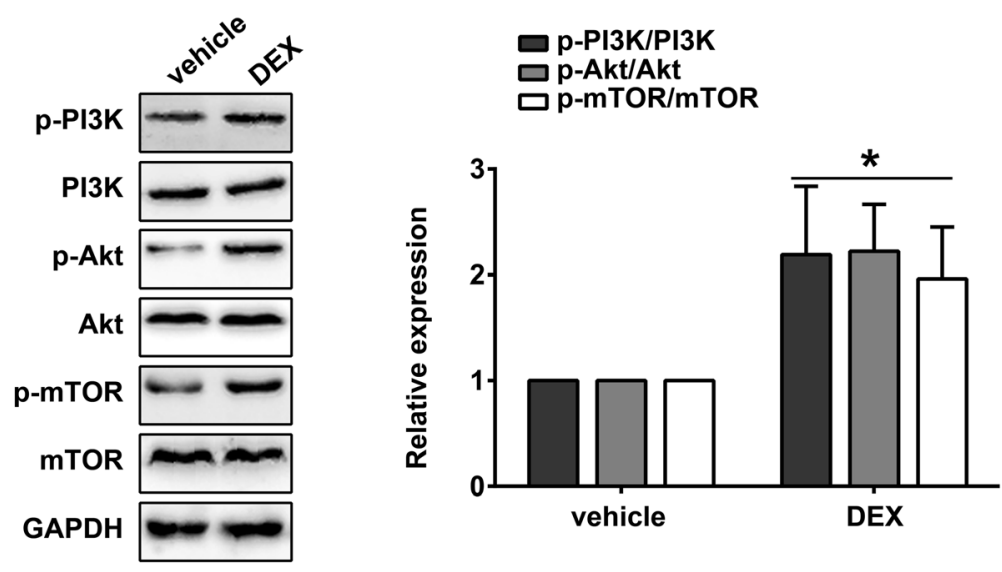

hippocampus
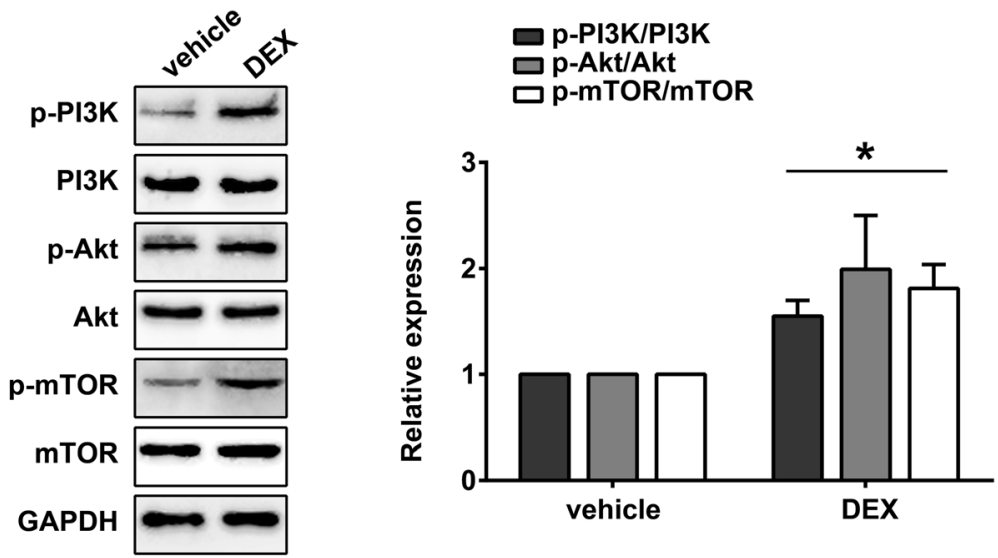

Fig. 4 Dexmedetomidine activated the PI3K/Akt/mTOR pathway in sevoflurane-treated rats Images and quantification of Western blot measurements of PI3K, p-PI3K, Akt, p-Akt, mTOR and p-mTOR in the cortex and hippocampus of rats in the vehicle and DEX groups (mean \pm SD, $n=5$ per group, $t$ test). ${ }^{*} p<0.05$ vs vehicle group

between sevoflurane anesthesia and neuroinflammation. We used low- or high-dose sevoflurane in rats, then detected the expression of proinflammatory cytokines (IL6 , IL-8, TNF- $\alpha$ ). We confirmed that sevoflurane could increase the levels of IL-6, IL- 8 and TNF- $\alpha$ in the cortex and hippocampus of anesthetized rats, in agreement with previous research which indicated that both isoflurane and sevoflurane were able to increase IL-6 levels via activation of NF- $\mathrm{kB}$ signalling [26].

The PI3K/Akt/mTOR cascade is important in mediating the release of proinflammatory cytokines [27-29]. PI3K is a ubiquitous lipid kinase which plays a crucial role in signal transduction through receptor tyrosine kinases. PI3K phosphorylates phosphatidylinositol-4,5-bis-phosphate (PIP2) to form phosphatidylinositol-4,5-tri-phosphate (PIP3); PIP3 recruits other downstream molecules, such as serine-threonine kinases, including the major effector of PI3K activation, Akt [30]. Activated Akt can then in turn activate mTOR, leading to the phosphorylation of two downstream effectors, p70 ribosomal protein S6 kinase 1 (S6K1) and eIF4E binding protein (4E-BP1), which promotes translational initiation and elongation [31-34]. In addition to its role in protein synthesis, mTOR also regulates the ubiquitin-proteasome system (UPS) [34, 35]. The UPS is an important regulator of NF- $\mathrm{KB}$ signaling, as the inhibitory regulator of NF- $\mathrm{kB}$ must be degraded by the proteasome in order for NF- $\mathrm{kB}$ to become active. Harston et al. suggested that inhibition of mTOR by rapamycin could increase polyubiquitination and ubiquitin-mediated degradation of ІкB$\alpha$, leading to NF- $\mathrm{KB}$-induced transcriptional activation. This result suggests that activation of mTOR could prevent the occurrence of inflammation through suppression of NF- $\mathrm{kB}$ activation [27], as NF- $\mathrm{kB}$ activity promotes the expression of proinflammatory cytokines [28, 29]. We observed an 


\section{cortex}
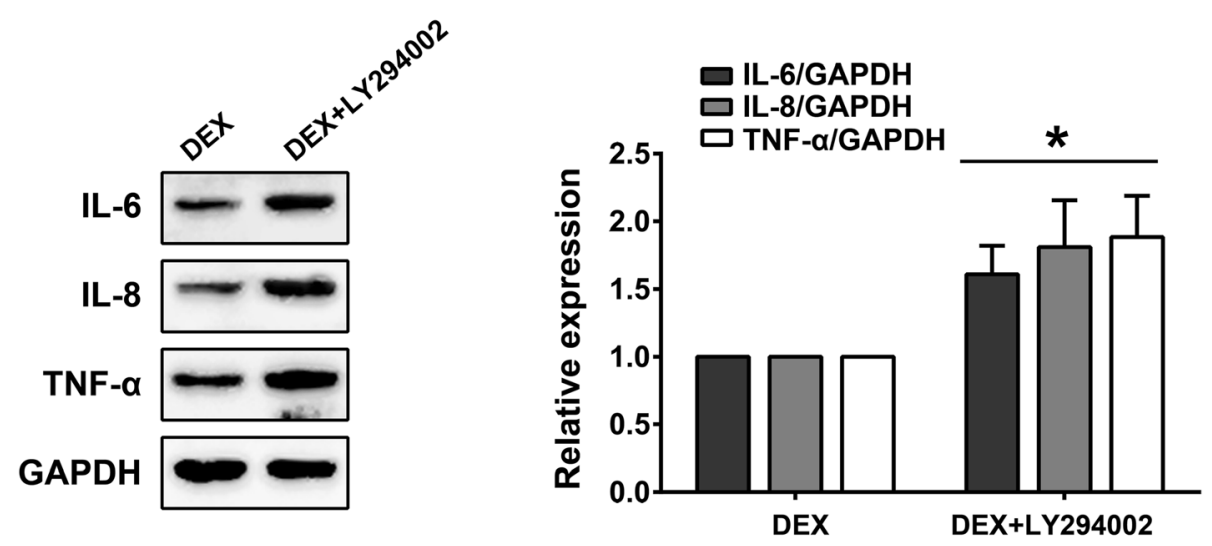

hippocampus
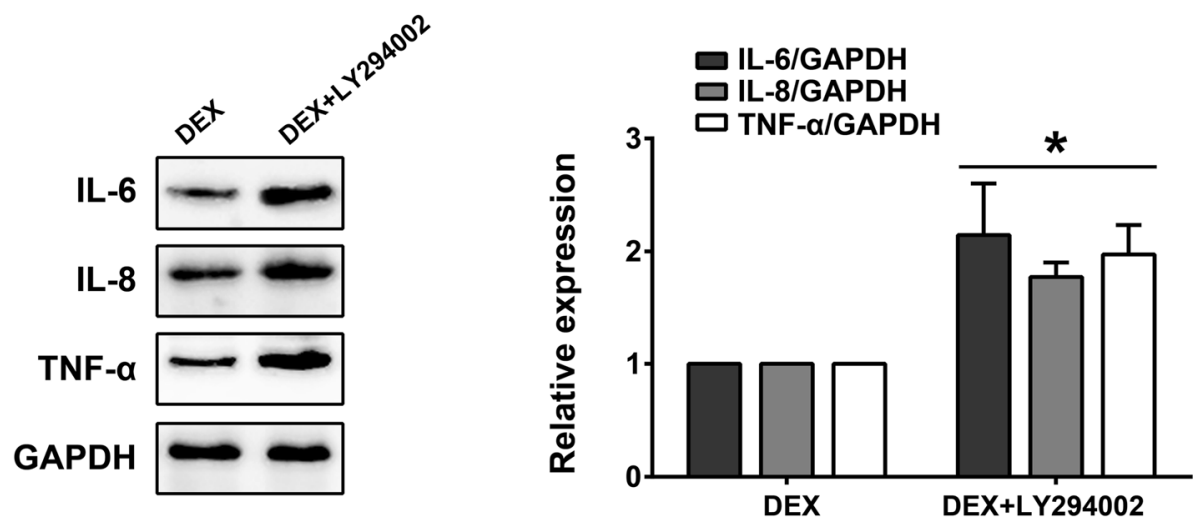

Fig. 5 Blockade of the PI3K/Akt/mTOR pathway reduced the anti-inflammatory activity of dexmedetomidine Western blot measurements of IL-6, IL-8 and TNF- $a$ from cortical and hippocampal samples of rats in the DEX and DEX + LY294002 groups (mean \pm SD, $n=5$ per group, t test). ${ }^{*} p<$ 0.05 vs DEX group

increase in pro-inflammatory cytokine release after treatment with sevoflurane anesthesia, and this release was accompanied by the inactivation of the PI3K/Akt/mTOR pathway, suggesting that inactivation of the PI3K/Akt/ mTOR pathway may be associated with sevoflurane anesthesia-induced neuroinflammation. Thus, increasing PI3K/Akt/mTOR pathway activity may be a promising and novel therapeutic strategy against postoperative neuroinflammation.

Recent studies have suggested that DEX has anti-inflammatory properties. Chen et al., for example, found that DEX treatment could suppress retinal ischemia/reperfusion injury, and showed effective anti-inflammatory effects through inhibition of toll-like receptor 4 (TLR4)/NF-kB expression [36]. Additionally, Huang et al. also suggested that DEX could inhibit the nuclear translocation and binding activity of activated $N F-k B$, thus reducing the release of inflammatory cytokines [37]. Given the anti-inflammatory properties of DEX and its effects on the PI3K/Akt/ mTOR signaling pathway, we hypothesized that DEX could suppress sevoflurane anesthesia-induced neuroinflammation. We found that treatment with DEX downregulated pro-inflammatory cytokines (IL-6, IL-8 and TNF- $\alpha$ ) and up-regulated the phosphorylation levels of PI3K/Akt/mTOR. A previous study indicated that DEX exerts an anti-inflammatory effect via the activation of $\mathrm{PI} 3 \mathrm{~K} / \mathrm{Akt} / \mathrm{mTOR}$ signaling in rats with traumatic brain injury [19]. In addition, blockade of the PI3K/Akt/mTOR pathway reduced the anti-inflammatory activity of DEX. Thus, we propose that DEX can ameliorate sevofluraneinduced neuroinflammation through activation of the PI3K/Akt/mTOR signaling pathway.

\section{Conclusions}

Sevoflurane anesthesia induces neuroinflammation in the CNS and this inflammation may be the result of decreased signaling through the PI3K/Akt/mTOR pathway. The administration of DEX can reduce neuroinflammation caused by sevoflurane, providing an important reference basis for clinical anesthesia. 


\section{Abbreviations}

DEX: Dexmedetomidine; NF-kB: Nuclear factor-kB pathway; PI3K/Akt/ mTOR: Phosphatidylinositol 3-hydroxy kinase/protein kinase B/mammalian target of rapamycin; PIP2: Phosphatidylinositol-4,5-bis-phosphate; PIP3: Phosphatidylinositol-4,5-tri-phosphate; S6K1: S6 kinase 1; SAH: Subarachnoid haemorrhage; TLR4: Toll like receptor 4; UPS: Ubiquitinproteasome system

\section{Acknowledgments}

We thank members of the laboratory for technical help.

\section{Authors' contributions}

The research idea and project design: MW. Data collection and dataset setup: NW. Data analysis: NW. All authors contributed to the development of the manuscript, and approved the final version.

\section{Funding}

No funding was received.

\section{Availability of data and materials}

The datasets used and/or analyzed during the current study are available from the corresponding author by reasonable request.

\section{Ethics approval and consent to participate}

All animal procedures were approved by the Institute of Radiation Medicine of the Chinese Academy of Medical Sciences and conducted in accordance with the ethical principles for Experiments on Animals, in addition to international standards.

\section{Consent for publication}

Not applicable.

\section{Competing interests}

The authors declare that they have no competing interests.

Received: 23 June 2019 Accepted: 18 July 2019

Published online: 27 July 2019

\section{References}

1. Tang L, Liu H, Wu Y, Li M, Li W, Jiang M, Hou J, Jiang Y, Xia Z, Meng Q. Sevoflurane may be more beneficial than propofol in patients receiving endoscopic variceal ligation and endoscopic variceal sclerotherapy: a randomized, double-blind study. Exp Ther Med. 2017;14:3145-52.

2. Huang L, Huang $\mathrm{K}$, Ning $\mathrm{H}$. Autophagy induction by hispidulin provides protection against sevoflurane-induced neuronal apoptosis in aged rats. Biomed Pharmacother. 2018:98:460-8.

3. Wang $L$, Zheng M, Wu S, Niu Z. MicroRNA-188-3p is involved in sevoflurane anesthesia-induced neuroapoptosis by targeting MDM2. Mo Med Rep. 2018;17:4229-36.

4. Tao G, Zhang J, Zhang L, Dong Y, Yu B, Crosby G, Culley DJ, Zhang Y, Xie Z. Sevoflurane induces tau phosphorylation and glycogen synthase kinase $3 \beta$ activation in young mice. Anesthesiology. 2014;121:510-27.

5. Liang P, Li F, Liu J, Liao D, Huang H, Zhou C. Sevoflurane activates hippocampal CA3 kainate receptors (Gluk2) to induce hyperactivity during induction and recovery in a mouse model. Br J Anaesth. 2017;119:1047-54.

6. Li D, Liu L, Li L, Li X, Huang B, Zhou C, Zhang Z, Wang C, Dong P, Zhang X, Yang B, Zhang L. Sevoflurane induces exaggerated and persistent cognitive decline in a type $\|$ diabetic rat model by aggregating hippocampal inflammation. Front Pharmacol. 2017:8:886.

7. Poor Zamany Nejat Kermany M, Roodneshin F, Ahmadi Dizgah N, Gerami E, Riahi E. Early childhood exposure to short periods of sevoflurane is not associated with later, lasting cognitive deficits. Paediatr Anaesth. 2016;26:1018-25.

8. Sun LS, Li G, Dimaggio C, Byrne M, Rauh V, Brooks-Gunn J, Kakavouli A, Wood A. Anesthesia and neurodevelopment in children: time for an answer. Anesthesiology. 2008:109:757-61.

9. Bo LJ, Yu PX, Zhang FZ, Dong ZM. Dexmedetomidine mitigates sevoflurane-induced cell cycle arrest in hippocampus. J Anesth. 2018; $32: 717-24$.
10. Kim BS, Choi JH, Baek SH, Lee DH. Effects of Intraneural injection of Dexmedetomidine in combination with Ropivacaine in rat sciatic nerve block. Reg Anesth Pain Med. 2018;43:378-84.

11. Brummett CM, Norat MA, Palmisano JM, Lydic R. Perineural administration of dexmedetomidine in combination with bupivacaine enhances sensory and motor blockade in sciatic nerve block without inducing neurotoxicity in rat. Anesthesiology. 2008;109:502-11.

12. Tan JA, Ho KM. Use of dexmedetomidine as a sedative and analgesic agent in critically ill adult patients: a meta-analysis. Intensive Care Med. 2010;36:926-39.

13. Alam A, Suen KC, Hana Z, Sanders RD, Maze M, Ma D. Neuroprotection and neurotoxicity in the developing brain: an update on the effects of dexmedetomidine and xenon. Neurotoxicol Teratol. 2017;60:102-16.

14. Wang L, Liu H, Zhang L, Wang G, Zhang M, Yu Y. Neuroprotection of Dexmedetomidine against cerebral ischemia-reperfusion injury in rats: involved in inhibition of NF-kB and inflammation response. Biomol Ther (Seoul). 2017;25:383-9

15. Yin D, Zhou S, Xu X, Gao W, Li F, Ma Y, Sun D, Wu Y, Guo Q, Liu H, Han L, Wang Z, Wang $Y$, Zhang J. Dexmedetomidine attenuated early brain injury in rats with subarachnoid haemorrhage by suppressing the inflammatory response: the TLR4/NF-KB pathway and the NLRP3 inflammasome may be involved in the mechanism. Brain Res. 2018;1698:1-10.

16. Qian XL, Zhang W, Liu MZ, Zhou YB, Zhang JM, Han L, Peng YM, Jiang JH, Wang QD. Dexmedetomidine improves early postoperative cognitive dysfunction in aged mice. Eur J Pharmacol. 2015;746:206-12.

17. Cui RS, Wang K, Wang ZL. Sevoflurane anesthesia alters cognitive function by activating inflammation and cell death in rats. Exp Ther Med. 2018:15:4127-30.

18. Yi C, Fu Z, Luo X. Dexmedetomidine on autophagy of hippocampal neurons in aged rats under sevoflurane anesthesia. Exp Ther Med. 2018;16:837-41.

19. Shen M, Wang S, Wen X, Han XR, Wang YJ, Zhou XM, Zhang MH, Wu DM, Lu J, Zheng YL. Dexmedetomidine exerts neuroprotective effect via the activation of the PI3K/Akt/mTOR signaling pathway in rats with traumatic brain injury. Biomed Pharmacother. 2017;95:885-93.

20. Umholtz M, Nader ND. Anesthetic immunomodulation of the Neuroinflammation in postoperative cognitive dysfunction. Immunol Investig. 2017:46:805-15.

21. Cortese GP, Burger C. Neuroinflammatory challenges compromise neuronal function in the aging brain: postoperative cognitive delirium and Alzheimer's disease. Behav Brain Res. 2017;322:269-79.

22. Pol RA, van Leeuwen BL, Izaks GJ, Reijnen MM, Visser L, Tielliu IF, Zeebregts CJ. C-reactive protein predicts postoperative delirium following vascular surgery. Ann Vasc Surg. 2014;28:1923-30.

23. Eger El. New inhaled anesthetics. Anesthesiology. 1994;80:906-22.

24. Esper $\mathrm{T}$, Wehner M, Meinecke CD, Rueffert $\mathrm{H}$. Blood/gas partition coefficients for isoflurane, sevoflurane, and desflurane in a clinically relevant patient population. Anesth Analg. 2015;120:45-50

25. Lee YM, Song BC, Yeum KJ. Impact of volatile anesthetics on oxidative stress and inflammation. Biomed Res Int. 2015;2015:242709.

26. Zhang L, Zhang J, Yang L, Dong Y, Zhang Y, Xie Z. Isoflurane and sevoflurane increase interleukin-6 levels through the nuclear factor-kappa B pathway in neuroglioma cells. Br J Anaesth. 2013;110(Suppl 1):i82-91.

27. Harston RK, McKillop JC, Moschella PC, Van Laer A, Quinones LS, Baicu CF, Balasubramanian S, Zile MR, Kuppuswamy D. Rapamycin treatment augments both protein ubiquitination and Akt activation in pressureoverloaded rat myocardium. Am J Physiol Heart Circ Physiol. 2011;300: H1696-706.

28. Tak PP, Firestein GS. NF-kappaB: a key role in inflammatory diseases. J Clin Invest. 2001;107:7-11.

29. Yang J, Li S, Wang L, Du F, Zhou X, Song Q, Zhao J, Fang R. Ginsenoside Rg3 attenuates lipopolysaccharide-induced acute lung injury via MerTK-dependent activation of the PI3K/AKT/mTOR pathway. Front Pharmacol. 2018;9:850.

30. Ko JK, Auyeung KK. Target-oriented mechanisms of novel herbal therapeutics in the chemotherapy of gastrointestinal cancer and inflammation. Curr Pharm Des. 2013:19:48-66.

31. Ma XM, Blenis J. Molecular mechanisms of mTOR-mediated translational control. Nat Rev Mol Cell Biol. 2009:10:307-18.

32. ladevaia V, Huo Y, Zhang Z, Foster LJ, Proud CG. Roles of the mammalian target of rapamycin, mTOR, in controlling ribosome biogenesis and protein synthesis. Biochem Soc Trans. 2012;40:168-72.

33. Albert $\mathrm{V}$, Hall MN mTOR signaling in cellular and organismal energetics. Curr Opin Cell Biol. 2015;33:55-66. 
34. Xu L, Brink M. mTOR, cardiomyocytes and inflammation in cardiac hypertrophy. Biochim Biophys Acta. 1863;2016:1894-903.

35. Zhang Y, Nicholatos J, Dreier JR, Ricoult SJ, Widenmaier SB, Hotamisligil GS, Kwiatkowski DJ, Manning BD. Coordinated regulation of protein synthesis and degradation by mTORC1. Nature. 2014;513:440-3.

36. Chen Z, Qiu PY, Ma CG. Dexmedetomidine preconditioning protects against retinal ischemia/reperfusion injury and inhibits inflammation response via toll-like receptor 4 (TLR4) pathway. Biomed Pharmacother. 2017;93:1018-24.

37. Huang Y, Lu Y, Zhang L, Yan J, Jiang J, Jiang H. Perineural dexmedetomidine attenuates inflammation in rat sciatic nerve via the NFKB pathway. Int J Mol Sci. 2014;15:4049-59.

\section{Publisher's Note}

Springer Nature remains neutral with regard to jurisdictional claims in published maps and institutional affiliations.

Ready to submit your research? Choose BMC and benefit from:

- fast, convenient online submission

- thorough peer review by experienced researchers in your field

- rapid publication on acceptance

- support for research data, including large and complex data types

- gold Open Access which fosters wider collaboration and increased citations

- maximum visibility for your research: over $100 \mathrm{M}$ website views per year

At BMC, research is always in progress.

Learn more biomedcentral.com/submissions 